\title{
Glutathione deficiency of the lower respiratory tract in patients with idiopathic pulmonary fibrosis
}

\author{
K.M. Beeh, J. Beier, I.C. Haas, O. Kornmann, P. Micke, R. Buhl
}

\begin{abstract}
Glutathione deficiency of the lower respiratory tract in patients with idiopathic pulmonary fibrosis. K. M. Beeh, J. Beier, I.C. Haas, O. Kornmann, P. Micke, R. Buhl. (C) ERS Journals Ltd 2002.

ABSTRACT: Idiopathic pulmonary fibrosis (IPF) is a disease of unknown aetiology. Increased oxidant burden and antioxidant, e.g. glutathione (GSH), deficiency in the lower respiratory tract have been thought to play a role in the progression of IPF. Sputum induction is a safe noninvasive tool to study inflammation in the respiratory tract.

The aim of the present study was to evaluate the direct measurement of GSH in induced sputum supernatant. Sixteen IPF patients and 15 healthy, nonsmoking subjects underwent sputum induction. Total GSH in sputum, saliva and plasma was measured spectrophotometrically.

Sputum GSH was decreased more then four-fold in IPF patients when compared to healthy subjects (mean GSH $1.4 \pm 0.34 \mu \mathrm{M}$ versus $5.8 \pm 0.98 \mu \mathrm{M}$ ). Salivary GSH was generally low or undetectable in all subjects. Plasma GSH levels were lower in IPF patients $(0.26 \pm 0.1$ versus $0.74 \pm 0.16 \mu M)$. In IPF patients, there was a borderline correlation of sputum GSH levels with disease duration and lung-function impairment.

These data confirm the established role of oxidant/antioxidant imbalance in the pathogenesis of idiopathic pulmonary fibrosis, and show the potential of induced sputum to directly study inflammatory processes and surrogate markers in interstitial lung diseases like idiopathic pulmonary fibrosis.

Eur Respir J 2002; 19: 1119-1123.
\end{abstract}

Pulmonary Dept, Internal Medicine, University Hospital Mainz, Mainz, Germany.

Correspondence: K.M. Beeh

Pulmonary Dept

Internal Medicine

University Hospital

Langenbeckstr. 1

55131 Mainz

Germany

Fax: 49161311175661

E-mail: k.beeh@3-med.klinik.

uni-mainz.de

Keywords: Antioxidants

glutathione

induced sputum

pulmonary fibrosis

Received: July 192001

Accepted after revision February 10 2002
Idiopathic pulmonary fibrosis (IPF) or cryptogenic fibrosing alveolitis is a chronic inflammatory disease of the lower respiratory tract leading to diffuse scarring of the lung parenchyma and end-stage tissue fibrosis $[1,2]$. The histological features of IPF usually comprise patterns of usual interstitial pneumonia, with irregular thickening of the alveolar septa, relatively mild tissue inflammation and patchy areas of heterogenous interstitial fibrosis [3]. Prognosis of IPF is poor, and pharmacotherapy with corticosteroids or immunosuppressive agents is of limited beneficial effect in these patients $[4,5]$. Therefore, there is an imminent need for new therapeutic strategies.

The cause of IPF is unknown and many of the pathomechanisms are poorly understood. Oxidative stress of the lower respiratory tract has been repeatedly linked to IPF, and an increased oxidant burden contributes to tissue remodelling and fibroblast activation in IPF and related disorders, like drug-induced pulmonary fibrosis [6-8].

The tripeptide glutathione (L-y-glutamyl-L-cysteinylglycine, GSH) plays a pivotal role in metabolic and cell-cycle related functions in virtually all cells. Its ability to directly scavenge free radicals and to act as a cosubstrate in the GSH peroxidase catalyzed reduction of $\mathrm{H}_{2} \mathrm{O}_{2}$ and lipid hydroperoxides makes
GSH central to defence mechanisms against intra- and extracellular oxidative stress [9].

Bronchoalveloar lavage (BAL) studies have shown, that IPF is characterized by GSH deficiency in the epithelial lining fluid [6, 10]. These findings raised hopes that therapeutic intervention with GSH or GSH precursors could delay the progression of the disease, and clinical studies using oral or inhaled GSH or $\mathrm{N}$-acetylcystein (NAC), a GSH precursor, have been performed or are currently underway $[11,12]$. A major problem faced by these studies is the evaluation of the therapeutic effect not only on clinical parameters, but also the target compartment, i.e. GSH levels in the respiratory tract, since this requires repeated BAL procedures in a large number of patients. Therefore, evaluation of GSH levels of the lower respiratory tract of IPF patients by means of induced sputum, a safe and reproducible tool that has been extensively studied in asthma or chronic obstructive pulmonary disease, has been assessed here.

\section{Methods}

\section{Patients}

The study population consisted of 16 patients (68\% males, mean age 59 yrs, range $37-72$ yrs) with 
a confirmed diagnosis of IPF according to the American Thoracic Society consensus report [13], and 15 healthy nonsmoking volunteers $(53 \%$ males, mean age $31 \mathrm{yrs}$, range $20-55 \mathrm{yrs}$ ). All patients with IPF were nonsmokers at the time of evaluation. Eleven IPF patients were treated with oral steroids (median dose $32 \mathrm{mg} \cdot \mathrm{day}^{-1}$, range $5-50 \mathrm{mg} \cdot$ day $^{-1}$ ), five received immunosuppressants (four azathioprin, one cyclophosphamide), and five were untreated. None of the IPF and control subjects was taking oral antioxidants, e.g. NAC. Pulmonary-function tests in IPF patients revealed a mean total lung capacity of $60 \pm 4 \%$ of predicted, and a diffusing capacity of $57 \pm 6 \%$ pred.

A venous blood sample was drawn from all subjects to measure plasma GSH levels.

\section{Sputum induction and processing}

Sputum induction was performed according to a method previously described [14], with slight modifications: patients inhaled $4 \%$ hypertonic saline delivered by an ultrasonic nebulizer device (UltraNeb 2000, DeVilbiss, UK) for $15 \mathrm{~min}$. Patients were then told to rinse their mouth, blow their nose and carefully cough sputum into a petri dish using forced expiratory manouevres. If the induction was well tolerated, the first portion of sputum was discarded, and the inhalation procedure was repeated for another $15 \mathrm{~min}$, resulting in a total induction time of $30 \mathrm{~min}$ for each individual patient. This was of particular importance, since initial experiments in healthy volunteers indicated, that the GSH concentration in sputum increased with induction length (data not shown). Lung function was carefully monitored by spirometry every $5 \mathrm{~min}$ during induction to ascertain safety of the procedure. Induction was stopped, when a $>20 \%$ drop in forced expiratory volume in one second (FEV1) occurred, and patients were given two puffs of salbutamol $\left(100 \mu \mathrm{g} \cdot \mathrm{puff}^{-1}\right)$.

To prevent mixing of sputum plugs with saliva and hence preventing diffusion of GSH from sputum to saliva, sputum plugs were immediately separated from salivary secretions, and closely examined by light microscopy. A 1-mL Eppendorf cup was filled with an appropriate sample, weighed, and mixed with twice the volume of phosphate-buffered saline (PBS). Although dithiotreitol (DTT) is used in standard protocols, none was added to the sputum sample, since preliminary experiments demonstrated a significant effect of DTT on GSH measurement in sputum (data not shown) and it acts as an antioxident itself [15]. Following addition of PBS, sputum was gently vortexed at room temperature until homogenization of the sample was achieved. Afterwards, samples were centrifuged at $400 \times g$ for $10 \mathrm{~min}$. The supernatant was then discarded and immediately processed for subsequent GSH measurement. The sputum sample was regarded as adequate when examination of cytospins by light microscopy revealed contamination with squamous cells of $<20 \%$. Simultaneously, all patients were asked to give a saliva sample, which was processed accordingly.
Measurement of total glutathione in plasma, sputum supernatant and saliva

Sputum GSH was measured using a spectrophotometrical method described previously [16]. Briefly, to determine total GSH levels (i.e. reduced GSH, GSH disulphide (GSSG)), plasma samples, saliva or sputum supernatant were mixed with an equal amount of $10 \mathrm{mM}$ 5,5'-dithiobis(2-nitrobenzoic acid) (DTNB)

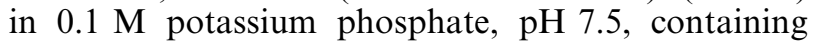
$17.5 \mathrm{mM}$ ethylenediaminetetraacetic acid (EDTA). The samples were centrifuged $(2,000 \times g, 10 \mathrm{~min})$, and aliquots $(50 \mu \mathrm{L})$ of the supernatants were added to cuvettes containing $0.5 \mathrm{U}$ of GSSG reductase in $0.1 \mathrm{M}$ potassium phosphate, $\mathrm{pH} \mathrm{7.5,} \mathrm{containing}$ $5 \mathrm{mM}$ EDTA. After incubation for $1 \mathrm{~min}$ at room temperature, the assay reaction was started by adding $220 \mathrm{nM}$ of reduced nicotinamide adenine dinucleotide phosphate in $0.1 \mathrm{M}$ potassium phosphate, $\mathrm{pH} 7.5$, containing $5 \mathrm{mM}$ EDTA in a final volume of $1 \mathrm{~mL}$. The rate of reduction of DTNB was recorded spectrophotometrically at a wavelength of $412 \mathrm{~nm}$ (Beckman DU-70 spectrophotometer; Beckman, Heidelberg, Germany). Determination of the total GSH concentration was based on standard curves generated from known concentrations of GSSG $(0.125-4 \mu \mathrm{M})$ in PBS, pH 7.4 .

\section{Statistical analysis}

Data are presented as mean \pm SEM. Group comparisons were performed with the Mann-Whitney U-test for non-normally distributed values, or t-test for normally distributed values. Correlations were calculated by Spearman's correlational analysis (rho). Since the disease duration was log normally distributed in the patients, $\log$ values (log months after diagnosis) were used for correlational analysis. A $p<0.05$ was considered statistically significant.

\section{Results}

Compared with healthy subjects, the total GSH content of the induced sputum supernatant from IPF patients was markedly reduced (mean GSH $1.4 \pm 0.34$ versus $5.8 \pm 0.98 \mu \mathrm{M}, \mathrm{p}<0.0011$ ) (fig. 1 ), with a fourfold decrease of the mean GSH concentration in IPF. Salivary GSH concentrations were generally low or undetectable in both groups $(p=0.2)$. Plasma GSH levels were lower in IPF patients $(0.26 \pm 0.1$ versus $0.74 \pm 0.16 \mu \mathrm{M}, \mathrm{p}=0.04)$, In IPF patients, there was an inverse correlation of sputum GSH levels with disease, with borderline significance duration (log months after diagnosis, rho=-0.45, $\mathrm{p}=0.07$ ) (fig. 2), and a positive correlation of GSH with lung function (vital capacity $\%$ pred, rho $=0.48, \mathrm{p}=0.05$ ) (fig. 3). However, no correlation of GSH with carbon monoxide diffusing capacity $\%$ pred was found $(r=0.31, p=0.24)$.

There was no correlation between plasma, salivary and sputum GSH levels in either group ( $p>0.1$, all correlations). 


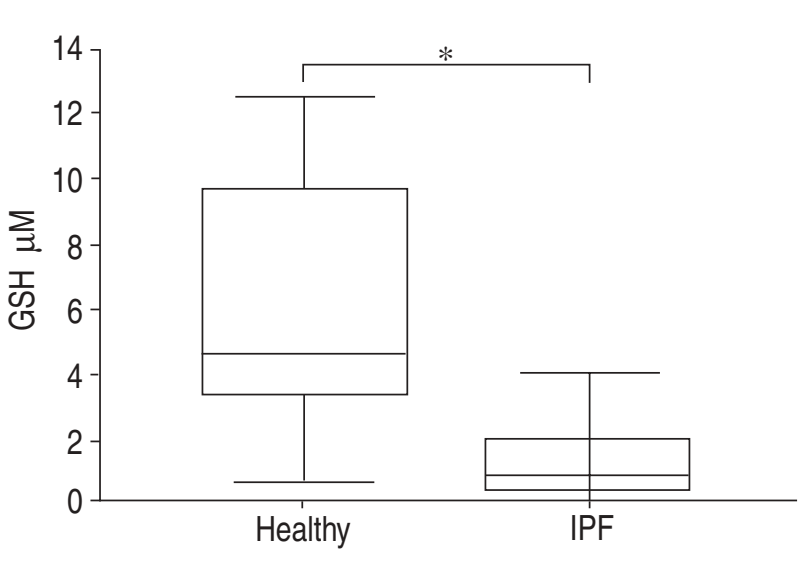

Fig. 1.-Box-Whisker plots of sputum glutathione (GSH) concentrations in idiopathic pulmonary fibrosis patients (IPF, $n=16)$ and healthy controls $(n=15){ }^{*}: \mathrm{p}<0.001$.

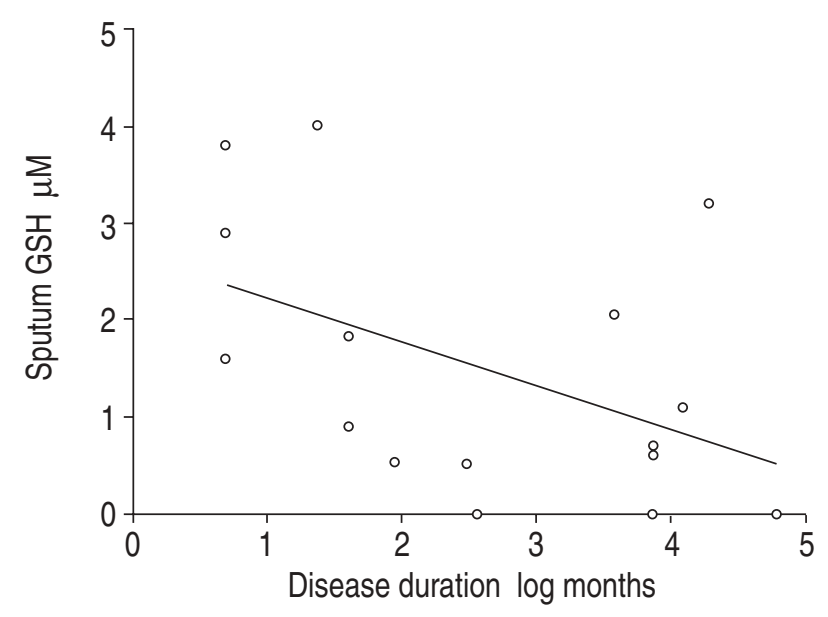

Fig. 2. - Correlation of sputum glutathione (GSH) concentrations and disease duration in idiopathic pulmonary fibrosis patients $(n=16)$.

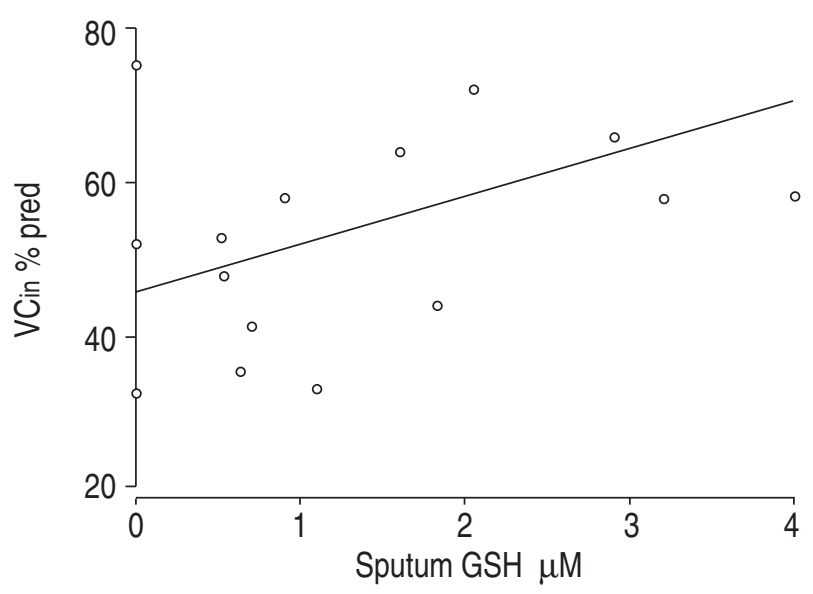

Fig. 3.-Correlation of sputum glutathione (GSH) concentrations and inspiratory vital capacity (VCin) in idiopathic pulmonary fibrosis patients $(n=16)$.

\section{Discussion}

IPF is a disease characterized by an increased oxidative burden in the lower respiratory tract of affected patients, and a marked deficiency of the major antioxidant GSH has been previously described in BAL studies with IPF patients [6]. Supplementation with antioxidants like NAC is therefore regarded a potential treatment option for IPF patients.

The present study focused on the possibility of measuring GSH concentrations in induced sputum from IPF patients. This is of particular interest, since the noninvasive procedure of sputum induction may offer a valuable tool to monitor GSH concentrations of the respiratory tract in clinical trials with antioxidant supplements, without the drawback of repeatedly exposing the patient to invasive and potentially harmful BAL procedures. Furthermore, despite a possible, albeit small, dilution effect resulting from inhalation of hypertonic saline, direct measurement of GSH in induced sputum supernatant avoids the necessity to calculate the sample dilution indirectly by evaluating the volume of the epithelial lining fluid, e.g. by the urea method.

The data obtained in this study revealed a marked deficiency of total GSH in induced sputum supernatant of IPF patients in comparison to healthy subjects. GSH levels of healthy subjects were fourfold higher than in IPF patients. This observation corresponds with BAL studies, that have reported a four-fold increase of the GSH content in the epithelial lung fluid of healthy subjects [6]. Nevertheless, the observed GSH concentrations in induced sputum of both patients and controls were markedly lower than the ones reported in BAL studies [6, 16, 17]. Few authors have studied the quantitative relationship between soluble and cellular markers in sputum or bronchial washing and BAL. For selected parameters, e.g. eosinophilic cationic protein or tryptase, a higher concentration in sputum than in BAL was found, whereas BAL contained more macrophages $[18,19]$. With regard to antioxidants, VAN DER VLIET et al. [20] were able to show a GSH gradient with low or undetectable concentrations in the nose, and high concentrations in BAL. This corresponds with another study in asthmatics, were BAL concentrations of GSH were markedly increased compared with bronchial washings [21]. GERSHMAn et al. [18] have demonstrated an increase in macrophages and surfactant protein A with increasing length of sputum induction, indicating that different lung compartments were sampled at different time points during sequential sputum induction. These findings are supported by experiments preliminary to this study, where a significant increase in sputum GSH was observed with rising induction length (saliva and sputum after 15, 30 and $45 \mathrm{~min}$ ) in healthy volunteers (data not shown). Similar to the evaluation of other markers in sputum, the use of strictly standardized induction protocols is crucial for the adequate interpretation of results. In this study, an induction time of $30 \mathrm{~min}$ was chosen, since this was tolerated best by all IPF patients and controls. Taken together, the results of this study suggest that there is a GSH 
gradient along the respiratory tract, with maximum concentrations in BAL. This finding is not surprising, due to the pivotal role of the alveolar epithelium as the primary antioxidative defence barrier [9]. Despite the fact that induced sputum contains mainly secretory products of midsized and small conducting airways, therefore not fully representative for the anatomic sites affected by IPF, this data strongly suggests that the magnitude of relative GSH deficiency in IPF can also be assessed by analysis of induced sputum supernatant.

In this study, the relative amount of oxidized GSH (GSSG, data not shown) could not be determined. Earlier BAL studies have shown that nearly the entire GSH found in BAL samples from IPF patients was present in the reduced form $[6,16]$. In contrast, BEHR et al. [22] have detected low concentrations of GSSG in native BAL fluid. However, the mean concentrations of total GSH were markedly higher in this study, suggesting that the impossibility to detect GSSG in induced sputum from the patients in this study is primarily a result of low sensitivity of the method at the lower detection limit, in particular, since sputum GSH was extremely low in the majority of IPF patients. CANTIN et al. [23], who encountered similar problems with detecting GSSG in BAL, speculated that due to the enrichment of the alveolar lining fluid with sulphydril-containing proteins, oxidative stress would favour the formation of mixed disulfphites, which would not be detected by the GSSG assay. It can be assumed, that this might also be true for sputum samples. Nevertheless, consistent with these previous observations in BAL, the basic finding of a GSH deficiency in this study would not have been affected.

Two general hypotheses have been established that might help explain the observation of a GSH deficiency in the lower respiratory tract of IPF patients: firstly, it was assumed that the inflammation of the lung parenchyma in IPF leads to an increased permeability of the alveolar epithelium, thus promoting a plasmatic dilution of GSH concentrations in BAL fluid [24]. Secondly, oxidative stress and increased oxidant burden by inflammatory cells involved in the pathogenesis of IPF deplete cellular sources of GSH, with a consecutive GSH deficiency [9, 16]. The present data support the second hypothesis, since it can be conceived, that the dilutory effect following an alveolar leak would not be of equal importance in induced sputum analyses. Furthermore, the data from this study suggest that GSH deficiency might develop as a consequence of alveolar and epithelial remodelling and fibrosis, since a negative correlation of sputum GSH levels with disease severity assessed by lung function and disease duration was observed.

Although in individual patients, there was no correlation between plasma and sputum GSH levels, the present data suggest that GSH-deficiency in the respiratory tract, similar to observations in human immunodeficiency virus-infected individuals [16], is at least partially due to a systemic GSH deficiency, since IPF patients also had significantly decreased plasma GSH levels. The observation of a decrease in plasma
GSH confirms findings of other studies demonstrating a reduced systemic antioxidant capacity in patients with pulmonary fibrosis [25]. This is of particular interest, since determination of plasma GSH could also serve as a simple method to monitor supplementation with antioxidants during therapeutic interventions.

To conclude, this study supports the concept of a contribution of oxidative stress and glutathione deficiency to the pathogenesis of idiopathic pulmonary fibrosis. To the best of the authors' knowledge, this is the first report on glutathione levels in induced sputum from idiopathic pulmonary fibrosis patients. In the future, induced sputum may provide a safe, noninvasive tool to monitor lower respiratory tract inflammation in interstitial lung diseases. Further studies evaluating the full potential of glutathione measurement in induced sputum, e.g. for prospective studies or serial assessments of patients under antioxidant supplementation are clearly warranted.

\section{References}

1. Crystal RG, Bitterman PB, Rennard SI, Hance AJ, Keogh BA. Interstitial lung diseases of unknown cause: disorders characterized by chronic inflammation of the lower respiratory tract. $N$ Eng J Med 1984; 310: 154-166 and 235-244.

2. Rochester CL, Elias JA. Cytokines and cytokine networking in the pathogenesis of interstitial and fibrotic lung disorders. Semin Respir Crit Care Med 1994; 14: 389-416.

3. King TE Jr. Idiopathic pulmonary fibrosis. In: Schwarz MI, King TE Jr, eds. Interstitial lung disease. St. Louis, Mosby, 1993; pp. 167-403.

4. Phan SH. New strategies for treatment of pulmonary fibrosis. Thorax 1995; 50: 415-421.

5. Hunninghake GW, Kalica AR. Approaches to the treatment of pulmonary fibrosis. Am J Respir Crit Care Med 1995; 151: 915-918.

6. Cantin AM, Hubbard RC, Crystal RG. Glutathione deficiency in the epithelial lining fluid of the lower respiratory tract in idiopathic pulmonary fibrosis. Am Rev Respir Dis 1989; 139: 370-372.

7. MacNee W, Rahman I. Oxidants/antioxidants in idiopathic pulmonary fibrosis. Thorax 1995; 50: 553-558.

8. Cooper JA Jr, White DA, Matthay RA. Drug-induced pulmonary disease. Part 1: Cytotoxic drugs. Am Rev Respir Dis 1986; 133: 321-340.

9. Davis WB, Pacht ER. Extracellular antioxidant defenses. In: Crystal RG, West JB, eds. The lung. Scientific foundations. New York, Raven Press, 1991; pp. $1821-1828$.

10. Crystal RG. Oxidants and respiratory tract epithelial injury: pathogenesis and strategies for therapeutic intervention. Am J Med 1991; 91: Suppl. 3C, 39S-44S.

11. Borok Z, Buhl R, Grimes GJ, et al. Effect of glutathione aerosol on oxidant-antioxidant imbalance in idiopathic pulmonary fibrosis. Lancet 1991; 338: 215-216.

12. Meyer A, Buhl R, Kampf S, Magnussen H. Intravenous $\mathrm{N}$-acetylcysteine and lung glutathione of patients with pulmonary fibrosis and normals. Am J Respir Crit Care Med 1995; 152: 1055-1060. 
13. American Thoracic Society. Idiopathic pulmonary fibrosis diagnosis and treatment. Am J Respir Crit Care Med 2000; 161: 646-664.

14. Pizzichini E, Pizzichini MMM, Efthimiadis A, et al. Indices of airway inflammation in induced sputum: reproducibility and validity of cell and fluid-phase measurements. Am J Respir Crit Care Med 1996; 154: 308-317.

15. Cleland WW. Dithiothreitol, a new protective agent for SH groups. Biochemistry 1964; 3: 80-82.

16. Buhl R, Jaffe HA, Holroyd KJ, et al. Systemic glutathione deficiency in symptom-free HIVseropositive individuals. Lancet 1989; 2: 1294-1298.

17. Buhl R, Vogelmeier C, Crittenden M, et al. Augmentation of glutathione in the fluid lining the epithelium of the lower respiratory tract by directly administering glutathione aerosol. Proc Natl Acad Sci USA 1990; 87: 4603-4607.

18. Gershman NH, Liu H, Wong HH, Liu JT, Fahy JV. Fractional analysis of sequential induced sputum samples during sputum induction: evidence that different lung compartments are sampled at different time points. J Allergy Clin Immunol 1999; 104: 322-328.

19. Fahy JV, Wong H, Liu J, Boushey HA. Comparison of samples collected by sputum induction and bronchoscopy from asthmatic and healthy subjects. Am J Respir Crit Care Med 1995; 152: 53-58.

20. van der Wiet $\mathrm{V}, \mathrm{O}^{\prime}$ Neill CA, Cross CE, et al. Determination of low-molecular-mass antioxidant concentrations in human respiratory tract lining fluids. Am J Physiol 1999; 276: L289-L296.

21. Smith LJ, Houston M, Anderson J. Increased levels of glutathione in bronchoalveolar lavage fluid from patients with asthma. Am Rev Respir Dis 1993; 147: 1461-1464.

22. Behr J, Maier K, Degenkolb B, Krombach F, Vogelmeier C. Antioxidative and clinical effects of high-dose N-acetylcysteine in fibrosing alveolitis. Am J Respir Crit Care Med 1997; 156: 1897-1901.

23. Cantin AM, Hubbard RC, Crystal RG. Glutathione deficiency in the epithelial lining fluid of the lower respiratory tract in idiopathic pulmonary fibrosis. $\mathrm{Am}$ Rev Respir Dis 1989; 139: 370-372.

24. Jones JG, Royston D, Minty BD. Changes in alveolarcapillary barrier function in animals and humans. $\mathrm{Am}$ Rev Respir Dis 1983; 127: 51-59.

25. Rahman I, Skwarska E, Henry M, et al. Systemic and pulmonary oxidative stress in idiopathic pulmonary fibrosis. Free Radic Biol Med 1999; 27: 60-68. 